\title{
Improved critical Hardy inequality and Leray-Trudinger type inequalities in Carnot groups
}

\author{
VAN HoAng NGUYen
}

\begin{abstract}
In this paper, we prove an improvement of the critical Hardy inequality in Carnot groups. We show that this improvement is sharp and can not be improved. We apply this improved critical Hardy inequality together with the Moser-Trudinger inequality due to Balogh, Manfredi and Tyson (2003) to establish the Leray-Trudinger type inequalities which extend the inequalities of Psaradakis and Spector (2015) and Mallick and Tintarev (2018) to the setting of Carnot groups.

\section{Parannettu kriittinen Hardyn epäyhtälö ja Leray-Trudinger-tyyppisiä epäyhtälöitä Carnot'n ryhmissä}

Tiivistelmä. Tässä paperissa todistamme kriittisen Hardyn epäyhtälön parannetun version Carnot'n ryhmissä. Osoitamme, että tämä parannus on tarkka, eikä sitä voi parantaa. Tämän kriittisen Hardyn epäyhtälön sekä Baloghin, Manfredin ja Tysonin (2003) esittämän MoserinTrudingerin epäyhtälön sovelluksena johdamme Leray-Trudinger-tyyppisiä epäyhtälöitä, jotka yleistävät Psaradakiksen ja Spectorin (2015) sekä Mallickin ja Tintarevin (2018) epäyhtälöitä Carnot'n ryhmien asetelmaan.
\end{abstract}

\section{Introduction}

The main aim of this paper is to study several inequalities in the setting of Carnot group such as the critical Hardy inequality and the Leray-Trudinger type inequality. Let us first recall some relevant results in this subject. Rough speaking, a Carnot group is a connected, simply connected, nilpotent Lie group $G$ of dimension at least two with finite step. The class of Carnot groups includes the Euclidean spaces (the commutative groups), the Heisenberg groups, and the Heisenberg type groups as special cases. Let $G$ be a Carnot group of homogeneous dimension $Q$, and let $\nabla_{0}$ denote the horizontal gradient on $G$. For a domain $\Omega$ in $G$, we denote by $H W_{0}^{1, p}(\Omega)$, $p \geq 1$ the horizontal Sobolev space. We refer the readers to Section $\S 2$ for more detailed account on the terminologies and background on Carnot groups.

We start by recalling the Hardy inequality in the Euclidean space $\mathbb{R}^{n}$ with $n \geq 2$,

$$
\int_{\mathbb{R}^{n}}|\nabla u|^{p} d x \geq\left(\frac{n-p}{p}\right)^{p} \int_{\mathbb{R}^{n}} \frac{|u|^{p}}{|x|^{p}} d x
$$

for any $1<p<n$ and $u \in C_{0}^{\infty}\left(\mathbb{R}^{n}\right)$. Furthermore, the constant $\left(\frac{n-p}{p}\right)^{p}$ is sharp and never achieved. The Hardy inequality with the same best constant still holds if $\mathbb{R}^{n}$ is replaced by any domain containing the origin in its interior. In the case $p=n$, the weight $|x|^{-n}$ is much singular, so there does not exist any positive constant $c$ such

https://doi.org/10.54330/afm.112567

2020 Mathematics Subject Classification: Primary 22E30, 43A80, 46E35.

Key words: Improved critical Hardy inequality, Leray-Trudinger inequality, Carnot groups.

(c) 2022 The Finnish Mathematical Society 
that the above inequality holds. In this case, we have a critical Hardy inequality (see $[2,9,10,4,32])$. Let us denote by

$$
E_{1}(t)=1-\ln t, E_{2}(t)=\ln \left(e E_{1}(t)\right), \ldots, E_{k}(t)=\ln \left(e E_{k-1}(t)\right), \quad k \geq 2, t \in(0,1] .
$$

For any bounded domain $\Omega$ in $\mathbb{R}^{n}$ containing the origin, let us denote by $R_{\Omega}=$ $\sup _{x \in \Omega}|x|$. Then, the following inequality holds

$$
I_{n}[u, \Omega, R]=: \int_{\Omega}|\nabla u|^{n} d x-\left(\frac{n-1}{n}\right)^{n} \int_{\Omega} \frac{|u|^{n}}{|x|^{n} E_{1}^{n}\left(\frac{|x|}{R}\right)} d x \geq 0, \quad u \in C_{0}^{\infty}(\Omega)
$$

The constant $\left(\frac{n-1}{n}\right)^{n}$ is sharp and never attained. So, $I_{n}[u, \Omega, R]>0$ for any $u \in$ $C_{0}^{\infty}(\Omega) \backslash\{0\}$. There have been many improvements of the Hardy inequality (see $[9,10,20,25,11]$ and references therein). Let us recall the the following improved critical Hardy inequality due to Barbatis, Filippas and Tertikas (see [9, Theorem B])

$$
\begin{aligned}
\int_{\Omega}|\nabla u|^{n} d x \geq & \left(\frac{n-1}{n}\right)^{n} \int_{\Omega} \frac{|u|^{n}}{|x|^{n} E_{1}^{n}\left(\frac{|x|}{R}\right)} d x \\
& +\frac{1}{2}\left(\frac{n-1}{n}\right)^{n-1} \sum_{k=2}^{\infty} \int_{\Omega} \frac{|u|^{n}}{|x|^{n} E_{1}^{n}\left(\frac{|x|}{R}\right) E_{2}^{2}\left(\frac{|x|}{R}\right) \cdots E_{k}^{2}\left(\frac{|x|}{R}\right)} d x
\end{aligned}
$$

for any $u \in C_{0}^{\infty}(\Omega)$. The constant $\frac{1}{2}\left(\frac{n-1}{n}\right)^{n-1}$ in the right-hand side of (1.2) is the best possible.

There have been many generalizations of the Hardy inequality in many different setting. The Hardy inequality in the Carnot groups was previously studied in [44, $45,43,12,18,19,24,26,27]$ and references therein. Let $u_{Q}$ be a singular solution of the sub-elliptic $Q$-Laplace on $G$ with pole at 0 (see Section $\S 2$ for more details on the existence and properties of $\left.u_{Q}\right)$. There exists $a_{Q}>0$ such that $N(x)=e^{-a_{Q} u_{Q}(x)}$ is a homogeneous norm on $G$. Let $\Omega$ be a bounded domain in $G$ containing the origin and set $R_{G, \Omega}:=\sup _{x \in \Omega} N(x)$. Then we have the following inequality which extends the critical Hardy inequality (1.1) to Carnot groups

$$
\begin{aligned}
& I_{G}[u, \Omega, R] \\
& :=\int_{\Omega}\left|\nabla_{0} u\right|^{Q} d x-\left(\frac{Q-1}{Q}\right)^{Q} \int_{\Omega}\left|\nabla_{0} N(x)\right|^{Q} \frac{|u(x)|^{Q}}{N(x)^{Q} E_{1}^{Q}\left(\frac{N(x)}{R}\right)^{Q}} d x \geq 0,
\end{aligned}
$$

for any $u \in C_{0}^{\infty}(\Omega)$ and $R \geq R_{G, \Omega}$. Here, the integral in (1.3) is taken with respect to the Haar measure on $G$. The constant $((Q-1) / Q)^{Q}$ is sharp and never attained. In the Heisenberg groups, the inequality (1.3) was established by Dou, Niu and Yuan [19].

The first main result of this paper provides an improvement of the inequality (1.3). More precisely, we have the following theorem. 
Theorem 1.1. Let $\Omega$ be bounded domain in $G$ containing the origin. Then for any $m \geq 2$, it holds

$$
\begin{aligned}
& \int_{\Omega}\left|\nabla_{0} u\right|^{Q} d x \geq\left(\frac{Q-1}{Q}\right)^{Q} \int_{\Omega}\left|\nabla_{0} N(x)\right|^{Q} \frac{|u(x)|^{Q}}{N(x)^{Q} E_{1}^{Q}\left(\frac{N(x)}{R}\right)^{Q}} d x \\
& \quad+\frac{1}{2}\left(\frac{Q-1}{Q}\right)^{Q-1} \sum_{k=2}^{m} \int_{\Omega}\left|\nabla_{0} N(x)\right|^{Q} \frac{|u(x)|^{Q}}{N(x)^{Q} E_{1}^{Q}\left(\frac{N(x)}{R}\right) \prod_{i=2}^{k} E_{i}^{2}\left(\frac{N(x)}{R}\right)} d x
\end{aligned}
$$

for any $u \in H W_{0}^{1, Q}(\Omega)$. Furthermore, for any $m \geq 2$, if there exists a positive constant $B>0$ for which the following inequality holds true

$$
\begin{aligned}
\int_{\Omega}\left|\nabla_{0} u\right|^{Q} d x & \geq\left(\frac{Q-1}{Q}\right)^{Q} \int_{\Omega}\left|\nabla_{0} N(x)\right|^{Q} \frac{|u(x)|^{Q}}{N(x)^{Q} E_{1}^{Q}\left(\frac{N(x)}{R}\right)^{Q}} d x \\
& +\frac{1}{2}\left(\frac{Q-1}{Q}\right)^{Q-1} \sum_{k=2}^{m-1} \int_{\Omega}\left|\nabla_{0} N(x)\right|^{Q} \frac{|u(x)|^{Q}}{N(x)^{Q} E_{1}^{Q}\left(\frac{N(x)}{R}\right) \prod_{i=2}^{k} E_{i}^{2}\left(\frac{N(x)}{R}\right)} d x \\
& +B \int_{\Omega}\left|\nabla_{0} N(x)\right|^{Q} \frac{|u(x)|^{Q}}{N(x)^{Q} E_{1}^{Q}\left(\frac{N(x)}{R}\right) \prod_{i=2}^{m-1} E_{i}^{2}\left(\frac{N(x)}{R}\right) E_{m}^{\gamma}\left(\frac{N(x)}{R}\right)} d x,
\end{aligned}
$$

for any $u \in H W_{0}^{1, Q}(\Omega)$ and for some $\gamma \in \mathbb{R}$, then we have

- $\gamma \geq 2$,

- and if $\gamma=2$ then $B \leq \frac{1}{2}\left(\frac{Q-1}{Q}\right)^{Q-1}$.

Consequently, the constant $\frac{1}{2}\left(\frac{Q-1}{Q}\right)^{Q-1}$ in the right-hand side of (1.4) is sharp.

Theorem 1.1 extends Theorem B and Proposition 3.2 in [9] (and hence extends the inequality (1.2) to the setting of Carnot groups.

The next result in this paper concerns with the Moser-Trudinger inequality in Carnot groups. Let $\Omega$ be a bounded domain in $\mathbb{R}^{n}$. The Moser-Trudinger inequality is the borderline case of the Sobolev inequality. It was shown independently by Yudovič [50], Pohožaev [40] and Trudinger [48] and sharpened later by Moser [37]

$$
\sup _{u \in W_{0}^{1, n}(\Omega),\|\nabla u\|_{L^{n}(\Omega)} \leq 1} \int_{\Omega} e^{\alpha|u|^{\frac{n}{n-1}}} d x<\infty,
$$

for any $\alpha \leq \alpha_{n}:=n \omega_{n-1}^{\frac{1}{n-1}}$ where $\omega_{n-1}$ denotes the surface area of the unit sphere in $\mathbb{R}^{n}$. Furthermore, the constant $\alpha_{n}$ is sharp in the sense that the left-hand side of (1.6) will become infinite if $\alpha>\alpha_{n}$. The Moser-Trudinger inequality plays the role of the Sobolev inequality in the borderline case. There have been many generalizations and improvements of the Moser-Trudinger inequality in literature (see $[1,3,5,8,16$, $15,14,29,30,31,33,42,35,47,49,46]$ and references therein). We also refer the readers to the papers $[13,21,34,17]$ for the results on the existence of extremals for the Moser-Trudinger inequality.

The Moser-Trudinger inequality in Carnot groups was proved by Balogh, Manfredi and Tyson [8]. They proved the existence of a constant $A_{Q}$ such that for any 
bounded domain $\Omega$ in $G$

$$
\sup _{u \in H W_{0}^{1, Q}(\Omega),\left\|\nabla_{0} u\right\|_{L} Q_{(\Omega)} \leq 1} \int_{\Omega} e^{\alpha|u|^{\frac{Q}{Q-1}}} d x<\infty,
$$

for any $\alpha \leq A_{Q}$, and if $\alpha>A_{Q}$ then the supremum in (1.7) will become infinite. Moreover, the constant $A_{Q}$ is given in terms of an integral on the unit sphere of the horizontal gradient of a certain homogeneous norm on $G$ (see (2.3) below). In the case of Heisenberg groups or the Heisenberg type groups (or H-groups), the sharp constant $A_{Q}$ was earlier found and explicitly computed by Cohn and Lu $[15,16]$ (see also $[14,31,29,49]$ for several other inequalities on Heisenberg groups with weights). The proof of (1.7) given in [8] follows the idea in [15] which is based on an representation formula via fundamental solution for the $Q$-Laplacian in Carnot groups, the O'Neil lemma [39] for the rearrangement of the convolution of two functions in Carnot groups, and the Adams' lemma [1, Lemma 1]. We refer the reader to [46] for an earlier result of Saloff-Coste on the Moser-Trudinger inequality on Carnot groups without sharp exponent.

Following the original Trudinger's approach [48], Psaradakis and Spector [41] proved the exponential integrability for functions in Euclidean space under the condition $I_{n}\left[u, \Omega, R_{\Omega}\right] \leq 1$. In fact, they proved a Leray-Trudinger inequality as follows: for any $\epsilon>0$, there exist positive constants $A_{n, \epsilon}$ depending only on $n, \epsilon$ and $B_{n}$ depending only on $n$ such that for any $0<c \leq A_{n, \epsilon}$

$$
\sup _{u \in C_{0}^{\infty}(\Omega), I\left[u, \Omega, R_{\Omega}\right] \leq 1} \int_{\Omega} \exp \left(c\left(\frac{|u(x)|}{E_{1}^{\epsilon}\left(\frac{|x|}{R_{\Omega}}\right)}\right)^{\frac{n}{n-1}}\right) d x \leq B_{n}|\Omega|
$$

and if $\epsilon=0$ then the supremum in (1.8) is infinite for any $c>0$. The inequality (1.8) was recently improved by Mallick and Tintarev [36] by proving that for any $\beta \geq \frac{2}{n}$ and $R \geq R_{\Omega}$, there exist positive constants $A_{n}$ and $B_{n}$ depending only on $n$ such that for any $0<c \leq A_{n}$

$$
\sup _{u \in C_{0}^{\infty}(\Omega), I[u, \Omega, R] \leq 1} \int_{\Omega} \exp \left(c\left(\frac{|u(x)|}{E_{2}^{\beta}\left(\frac{|x|}{R_{\Omega}}\right)}\right)^{\frac{n}{n-1}}\right) d x \leq B_{n}|\Omega|,
$$

and if $\beta<\frac{1}{n}$ then the above supremum is $+\infty$. The Leray-Trudinger type inequalities (1.8) and (1.9) are extended to higher order Sobolev spaces in [38] by the author.

The next main result is an extension of the inequality (1.9) to the context of Carnot groups.

Theorem 1.2. Let $\Omega$ be a bounded domain in $G$ containing the origin. Then for any $\beta \geq \frac{2}{Q}$ and $R \geq R_{G, \Omega}$, there exist constants $A_{Q, N}$ depending on $Q, N$ and $B_{Q}$ depending on $Q$ such that for any $0<c<A_{Q, N}$, it holds

$$
\sup _{u \in H W_{0}^{1, Q}(\Omega), I_{G}[u, \Omega, R] \leq 1} \int_{\Omega} \exp \left(c\left(\frac{|u|}{E_{2}^{\beta}\left(\frac{N(x)}{R}\right)}\right)^{\frac{Q}{Q-1}}\right) d x \leq B_{Q}|\Omega| .
$$

Moreover, if $\beta<\frac{1}{Q}$ the inequality (1.10) fails in the sense that for any $c>0$ the supremum in (1.10) is infinite. 
The explicit expressions of $A_{Q, N}$ and $B_{Q}$ can be found in the proof of Theorem 1.2. More precisely, we have

$$
A_{Q, N}=2^{-\frac{Q-1}{Q}}\left(C_{1}(Q)+2 \frac{Q}{Q-1}\right)^{-\frac{1}{Q}} A_{Q}
$$

with $A_{Q}$ being the sharp exponent in (1.7) and $B_{Q}$ is the supremum in (1.7). As an immediate consequence, we obtain the following extension of the inequality (1.8) due to Psaradakis and Spector to Carnot groups,

Corollary 1.3. Let $\Omega$ be a bounded domain in $G$ containing the origin. Then for any $\epsilon>0$, there exist positive constants $A_{Q, N, \epsilon}$ depending only on $Q, N, \epsilon$ and $B_{Q}$ depending only on $Q$ such that for any $0<c \leq A_{Q, N, \epsilon}$

$$
\sup _{u \in H W_{0}^{1, Q}(\Omega), I_{G}[u, \Omega, R] \leq 1} \int_{\Omega} \exp \left(c\left(\frac{|u(x)|}{E_{1}^{\epsilon}\left(\frac{N(x)}{R_{\Omega}}\right)}\right)^{\frac{Q}{Q-1}}\right) d x \leq B_{Q}|\Omega| .
$$

Moreover, such an estimate fails when $\epsilon=0$.

The proof of Theorem 1.2 uses some ideas from [41, 36]. Indeed, we first make a ground state transform $u(x)=E_{1}^{\frac{Q-1}{Q}}\left(\frac{N(x)}{R}\right) v(x)$ and show that

$$
I_{G}[u, \Omega, R] \geq C_{1}(Q) \int_{\Omega} E_{1}^{Q-1}\left(\frac{N(x)}{R}\right)\left|\nabla_{0} v\right|^{Q} d x .
$$

Doing the simple computations, we get

$$
\begin{aligned}
& \left|\nabla_{0}\left(\frac{u(x)}{E_{2}^{\frac{2}{Q}}\left(\frac{N(x)}{R}\right)}\right)\right| \\
& \leq\left|\nabla_{0} v(x)\right| E_{1}^{\frac{Q-1}{Q}}\left(\frac{N(x)}{R}\right)+\frac{Q-1}{Q} \frac{\left|\nabla_{0} N(x)\right|}{N(x)} \frac{|u(x)|}{E_{1}\left(\frac{N(x)}{R}\right) E_{2}^{\frac{2}{Q}}\left(\frac{N(x)}{R}\right)}
\end{aligned}
$$

The ground state transform above together with the improved critical Hardy inequality (Theorem 1.1) show that the $L^{Q}$-norm of the right-hand side is uniformly bounded. Hence, the inequality 1.10 would follows from (1.7). The second statement is also a consequence of Theorem 1.1 .

\section{Preliminaries}

In this section, we briefly recall some facts related to analysis in Carnot groups. A Carnot group is a connected, simply connected, nilpotent Lie group $G$ of dimension at least two with graded Lie algebra $\mathfrak{g}=V_{1} \oplus \cdots \oplus V_{r}$ so that $\left[V_{1}, V_{i}\right]=V_{i+1}$ for $i=1, \ldots, r-1$ and $\left[V_{1}, V_{r}\right]=0$ here $[\cdot, \cdot]$ denotes the Lie bracket on $\mathfrak{g}$. The number $r \geq 1$ is called the step of $G$. We denote the neutral element of $G$ by 0 and we identify the elements of $\mathfrak{g}$ with left-invariant vector fields on $G$ in the usual manner.

We fix throughout this paper an scalar product $\langle\cdot, \cdot\rangle_{0}$ in $V_{1}$ associated with an orthonormal basis $X_{1}, \ldots, X_{k}$. Relative this basis, we construct the horizontal tangent subbundle $H T G$ of the tangent bundle $T G$ with fibers $H T_{x} G$ which is the vector space spanned by $X_{1}(x), \ldots, X_{k}(x), x \in G$. A left-invariant vetor field $X$ on $G$ is said to be horizontal if it is a section of the horizontal tangent bundle. 
The group $G$ is diffeomorphic with its algebra $\mathfrak{g}=\mathbb{R}^{m}$ with $m=\sum_{i=1}^{r} \operatorname{dim} V_{i}$ via the exponential map exp $: \mathfrak{g} \rightarrow G$. Hence, we can identify an element $g \in G$ with an element $x=\left(x_{1}, \ldots, x_{k}, t_{k+1}, \ldots, t_{m}\right) \in \mathbb{R}^{m}$ by the formula

$$
g=\exp \left(\sum_{i=1}^{k} x_{i} X_{i}+\sum_{i=k+1}^{m} t_{i} T_{i}\right)
$$

where $T_{k+1}, \ldots, T_{m}$ denote a set of non-horizontal vectors extending $X_{1}, \ldots, X_{k}$ to a basis of $\mathfrak{g}$.

The Haar measure on $G$ is induced by the exponential map from the Lebesgue measure on $\mathfrak{g}=\mathbb{R}^{m}$. Throughout this paper, statements involving measure theory are always understood to be with respect to Haar measure.

The horizontal divergence of a vector field

$$
\eta=\sum_{i=1}^{k} \varphi_{i} X_{i}+\sum_{i=k+1}^{m} \psi_{i} T_{i}
$$

is given by

$$
\operatorname{div}_{0} \eta=\sum_{i=1}^{k} X_{i}\left(\varphi_{i}\right) .
$$

Let $U$ be a domain of $G$. For a function $f \in L_{\text {loc }}^{1}(U)$ the space of locally integrable functions on $U$, we say that the horizontal gradient of $f$ exists in the distributional sense if there exists a horizontal vector field $v=\sum_{i=1}^{k} v_{i} X_{i}$ with $v_{i} \in L_{\text {loc }}^{1}(U)$ such that

$$
\int_{U}\langle v, \eta\rangle_{0} d x=-\int_{U} f \operatorname{div}_{0} \eta d x
$$

for any smooth compactly supported horizontal vector fields $\eta$ in $U$. We write $\nabla_{0} f=$ $v$ for the horizontal gradient of $f$. Note that if $f \in C^{1}(U)$ then $\nabla_{0} f$ is the unique horizontal vector field in $U$ defined by the equation

$$
\left\langle\nabla_{0} f, X\right\rangle_{0}=X(f)
$$

for any horizontal vector fields $X$ in $U$. For $p \geq 1$, we denote by $H W^{1, p}(U)$ the horizontal Sobolev space consisting all function $f \in L^{p}(U)$ such that $\nabla_{0} f$ exists in the distributional sense and $\left|\nabla_{0} f\right| \in L^{p}(U)$ where $|\cdot|$ is the norm on $V_{1}$ induced by the scalar product $\langle\cdot, \cdot\rangle_{0} . H W_{0}^{1, p}(U)$ is the closure of $C_{0}^{\infty}(\Omega)$ in $H W^{1, p}(\Omega)$.

For $t>0$, the one-parameter family of dilatations $\left\{\delta_{t}\right\}_{t}$ acting on $\mathfrak{g}$ is defined by $\delta_{t}(X)=t^{i} X$ if $X \in V_{i}$ and extending by linearity. Via the exponential map, $\delta_{t}$ induces an automorphism of $G$ onto itself which we still denote by $\delta_{t}$. The Jacobian determinant of $\delta_{t}$ (relative to Haar measure) is $t^{Q}$ where

$$
Q=\sum_{i=1}^{r} i \operatorname{dim} V_{i}
$$

is the homogeneous dimension of $G$ which plays the role of dimension in analysis in Carnot groups.

A homogeneous norm on $G$ is a nonnegative function $f: G \rightarrow[0, \infty)$ so that

(i) $f(x)>0$ if $x \neq 0$ and $f(0)=0$,

(ii) $f\left(\delta_{t}(x)\right)=t f(x)$ for any $t>0$ and $x \in G$,

(iii) $f\left(x^{-1}\right)=f(x)$ for any $x \in G$. 
Suppose that $G$ is a Carnot group of homogeneous dimension $Q \geq 3$. Let $U$ be a domain in $G$ and $1<p<\infty$. A function $f \in H W^{1, p}(U)$ is said to be a (weak) solution to the sub-elliptic $p$-Laplace equation in $U$ if

$$
\int_{U}\left|\nabla_{0} f\right|^{p-2}\left\langle\nabla_{0} f, \nabla_{0} \phi\right\rangle_{0} d x=0
$$

for any test function $\phi \in C_{0}^{\infty}(U)$. In case $\left|\nabla_{0} f\right|^{p-2} \nabla_{0} f \in C^{1}(U)$, the standard method shows that (2.1) is equivalent to the equation

$$
\Delta_{0, p} f:=\operatorname{div}_{0}\left(\left|\nabla_{0} f\right|^{p-2} \nabla_{0} f\right)=\sum_{i=1}^{k} X_{i}\left(\left|\nabla_{0} f\right|^{p-2} X_{i} f\right)=0 .
$$

We call a function $f$ to be $p$-harmonic if it satisfies (2.1) in $U$. The operator $\Delta_{0, p}$ is called the sub-elliptic $p$-Laplace operator. For basis results on potential theory on Carnot groups, we refer the readers to $[28,12]$.

In the case $p=2$, we write $\Delta_{0}=\Delta_{0,2}=\sum_{i=1}^{k} X_{i}^{2}$. This is Kohn's sub-Laplace operator on $G$. The harmonic analysis associated with $\Delta_{0}$ has been a subject of many investigations. Folland [22] proved in any Carnot group that there exists a unique fundamental solution $u_{2}$ to the equation for the 2-Laplace operator which is smooth away from 0 and homogeneous of degree $2-Q$. For the non-linear case $p \neq 2$, there are existence results but there is no regularity theory for the solutions of the $p$-Laplace operator except for the special case of Heisenberg groups or H-type groups (see [7, 12, 28]). From [28, Proposition 4.16], there is a weak solution $u_{Q}$ of the $Q$-Laplace equation (the so-called singular solution) that is continuous on $G \backslash\{0\}$, has prescribed singularity $\lim _{x \rightarrow 0} u_{Q}(x)=\infty$ and $\lim _{x \rightarrow \infty} u_{Q}(x)=-\infty$. According to an additional result in [6], there exists a positive constant $a_{Q}>0$ such that the function $N(x)=\exp \left(-a_{Q} u_{Q}(x)\right)$ if $x \neq 0$ and $u(0)=0$ defined a homogeneous norm on $G$. Let $S$ denote the unit sphere with respect to $N$, i.e., $S=\{x: N(x)=1\}$. It was shown in [23] that there exists a Radon measure $\sigma$ on $S$ such that for any $f \in L^{1}(G)$

$$
\int_{G} f(x) d x=\int_{0}^{\infty} \int_{S} f\left(\delta_{s}(x)\right) d \sigma(x) s^{Q-1} d s .
$$

Note that $\left.\left|\nabla_{0} N\right|\right|_{S} \in L^{Q}(S, d \sigma)$ (see [8, Lemma 2.9]). Let us define

$$
c_{Q}=\int_{S}\left|\nabla_{0} N\right|^{Q} d \sigma
$$

The existence of the fundamental solution of the $Q$-Laplace equation was proved by Balogh, Manfredi and Tyson in [8, Theorem 3.1]. More precisely, they proved that up to a constant multiply $u_{Q}$ is a fundamental solution of the $Q$-Laplace equation, i.e., there is $b_{Q} \in \mathbb{R}$ such that

$$
-\operatorname{div}_{0}\left(\left|\nabla_{0} u_{Q}\right|^{Q-2} \nabla_{0} u_{Q}\right)=b_{Q} \delta_{0}
$$

They also show that

$$
\operatorname{div}_{0}\left(\frac{\left|\nabla_{0} N\right|^{Q-2}}{N^{Q-1}} \nabla_{0} N\right)=c_{Q} \delta_{0}
$$

Now for any $u \in C_{0}^{\infty}(\Omega \backslash\{0\})$, let us define the new function $v$ by

$$
v(x)=u(x) E_{1}^{-\frac{Q-1}{Q}}\left(\frac{N(x)}{R}\right) .
$$


We then have the following result:

Proposition 2.1. For any $R \geq R_{\Omega}$, it holds

$$
I_{G}[u, \Omega, R] \geq C_{1}(Q) \int_{\Omega} E_{1}^{Q-1}\left(\frac{N(x)}{R}\right)\left|\nabla_{0} v(x)\right|^{Q} d x .
$$

Proof. By the straightforward computations, we have

$$
\nabla_{0} u(x)=E_{1}^{\frac{Q-1}{Q}}\left(\frac{N(x)}{R}\right) \nabla_{0} v(x)-\frac{Q-1}{Q} E_{1}^{-\frac{1}{Q}}\left(\frac{N(x)}{R}\right) v(x) \frac{\nabla_{0} N(x)}{N(x)} .
$$

Using the elementary inequality (see [10, Lemma 3.1])

$$
|a+b|^{p} \geq|a|^{p}+p|a|^{p-2}\langle a, b\rangle+C_{1}(p)|b|^{p}, \quad p \geq 2, a, b \in \mathbb{R}^{n},
$$

we have

$$
\begin{aligned}
\left|\nabla_{0} u(x)\right|^{Q} \geq & \left(\frac{Q-1}{Q}\right)^{Q} \frac{|u(x)|^{Q}}{N(x)^{Q} E_{1}^{Q}\left(\frac{N(x)}{R}\right)|v(x)|^{Q}}\left|\nabla_{0} N(x)\right|^{Q} \\
& -\left(\frac{Q-1}{Q}\right)^{Q-1} \frac{\left\langle\nabla_{0}\left(|v(x)|^{Q}\right),\left|\nabla_{0} N(x)\right|^{Q-2} \nabla_{0} N(x)\right.}{N(x)^{Q-1}} \\
& +C_{1}(Q) E_{1}^{Q-1}\left(\frac{N(x)}{R}\right)\left|\nabla_{0} v(x)\right|^{Q} .
\end{aligned}
$$

Integrating the previous inequality in $G$ and using integration by parts and (2.4), we obtain the desired inequality.

\section{Proof of Theorem 1.1}

In this section, we prove Theorem 1.1. The proof follows the idea from [9] by using a suitable horizontal vector field.

Proof of Theorem 1.1. We define the horizontal vector field $T$ on $G$ by

$$
\begin{aligned}
T(x)= & \left(\frac{Q-1}{Q}\right)^{Q-1} \frac{\left|\nabla_{0} N(x)\right|^{Q-2} \nabla_{0} N(x)}{N(x)^{Q-1}}\left(E_{1}^{1-Q}\left(\frac{N(x)}{R}\right)\right. \\
& \left.+\sum_{i=2}^{m} E_{1}^{1-Q}\left(\frac{N(x)}{R}\right) E_{2}^{-1}\left(\frac{N(x)}{R}\right) \cdots E_{i}^{-1}\left(\frac{N(x)}{R}\right)\right) .
\end{aligned}
$$

Notice that

$$
E_{1}^{\prime}(t)=-\frac{1}{t}, \quad E_{k}^{\prime}(t)=-\frac{1}{t}\left(E_{1}(t) \cdots E_{k-1}(t)\right)^{-1}, \quad k \geq 2, t \in(0,1] .
$$

From (2.4) and (3.1), we can easily verify that

$$
\begin{aligned}
\operatorname{div}_{0} T(x)= & \frac{(Q-1)^{Q}}{Q^{Q-1}} \frac{\left|\nabla_{0} N(x)\right|^{Q}}{N(x)^{Q} E_{1}^{Q}\left(\frac{N(x)}{R}\right)}\left[1+\sum_{i=2}^{m} \frac{1}{E_{2}\left(\frac{N(x)}{R}\right) \cdots E_{i}\left(\frac{N(x)}{R}\right)}\right. \\
& \left.+\frac{1}{Q-1} \sum_{i=2}^{m} \sum_{j=2}^{i} \frac{1}{E_{2}^{2}\left(\frac{N(x)}{R}\right) \cdots E_{j}^{2}\left(\frac{N(x)}{R}\right) E_{j+1}\left(\frac{N(x)}{R}\right) \cdots E_{i}\left(\frac{N(x)}{R}\right)}\right]
\end{aligned}
$$


for a.e. $x \in G$. Using the estimates in the proof of [9, Theorem B], we have

$$
\begin{aligned}
\operatorname{div}_{0} T(x)-(Q-1)|T(x)|^{\frac{Q}{Q-1}} & \frac{(Q-1)^{Q}}{Q^{Q-1}} \frac{\left|\nabla_{0} N(x)\right|^{Q}}{N(x)^{Q} E_{1}^{Q}\left(\frac{N(x)}{R}\right)}\left[1+\sum_{i=2}^{m} \frac{1}{E_{2}\left(\frac{N(x)}{R}\right) \cdots E_{i}\left(\frac{N(x)}{R}\right)}\right. \\
& +\frac{1}{Q-1} \sum_{i=2}^{m} \sum_{j=2}^{i} \frac{1}{E_{2}^{2}\left(\frac{N(x)}{R}\right) \cdots E_{j}^{2}\left(\frac{N(x)}{R}\right) E_{j+1}\left(\frac{N(x)}{R}\right) \cdots E_{i}\left(\frac{N(x)}{R}\right)} \\
& \left.-\frac{Q-1}{Q}\left(1+\sum_{i=2}^{m} \frac{1}{E_{2}\left(\frac{N(x)}{R}\right) \cdots E_{i}\left(\frac{N(x)}{R}\right)}\right)^{\frac{Q}{Q-1}}\right] \\
\geq & \left(\frac{Q-1}{Q}\right)^{Q} \frac{\left|\nabla_{0} N(x)\right|^{Q}}{N(x)^{Q} E_{1}^{Q}\left(\frac{N(x)}{R}\right)}\left(1+\frac{Q}{2(Q-1)} \sum_{i=2}^{m} \frac{1}{E_{2}^{2}\left(\frac{N(x)}{R}\right) \cdots E_{i}^{2}\left(\frac{N(x)}{R}\right)}\right)
\end{aligned}
$$

for a.e. $x \in G$. Multiplying both sides by $|u(x)|^{Q}$ and integrating the obtained inequality in $G$, we get

$$
\begin{aligned}
& \left(\frac{Q-1}{Q}\right)^{Q} \int_{G} \frac{\left|\nabla_{0} N(x)\right|^{Q}}{N(x)^{Q} E_{1}^{Q}\left(\frac{N(x)}{R}\right)}\left(1+\frac{Q}{2(Q-1)} \sum_{i=2}^{m} \frac{1}{E_{2}^{2}\left(\frac{N(x)}{R}\right) \cdots E_{i}^{2}\left(\frac{N(x)}{R}\right)}\right) \\
& \quad \times|u(x)|^{Q} d x \leq \int_{G}|u(x)|^{Q} \operatorname{div}_{0} T(x) d x-(Q-1) \int_{G}|u(x)|^{Q}|T(x)|^{\frac{Q}{Q-1}} d x .
\end{aligned}
$$

Using integration by parts and Hölder inequality, we obtain

$$
\begin{aligned}
\int_{G}|u(x)|^{Q} \operatorname{div}_{0} T(x) d x & =-Q \int_{G}|u(x)|^{Q-2} u(x)\left\langle\nabla_{0} u(x), T(x)\right\rangle_{0} d x \\
& \leq \int_{G}\left|\nabla_{0} u(x)\right|^{Q} d x+(Q-1) \int_{G}|u(x)|^{Q}|T(x)|^{\frac{Q}{Q-1}} d x .
\end{aligned}
$$

Inserting (3.3) into (3.2) we get the desired inequality (1.4).

Suppose that the inequality (1.5) holds true for some $B>0, \gamma \in \mathbb{R}$. Without loss of generality, we assume that the unit ball $B_{N}:=\{x: N(x)<1\}$ is included in $\Omega$. Let $\psi \in C^{\infty}([0, \infty))$ be such that $\psi(t)=1$ if $t \leq \frac{1}{2}, \psi(t)=0$ if $t \geq 1$ and $0 \leq \psi \leq 1$. Define $\varphi(x)=\psi(N(x))$. For any $t>0$, denote $\varphi_{t}(x)=\varphi\left(\delta_{\frac{1}{t}}(x)\right)$.

For small parameters $\alpha_{1}, \ldots, \alpha_{m}>0$, we define

$$
w(x)=E_{1}^{\frac{Q-1-\alpha_{1}}{Q}}\left(\frac{N(x)}{R}\right) E_{2}^{\frac{1-\alpha_{2}}{Q}}\left(\frac{N(x)}{R}\right) \cdots E_{m}^{\frac{1-\alpha_{m}}{Q}}\left(\frac{N(x)}{R}\right),
$$

and

$$
u(x)=\varphi(x) w(x) .
$$


We claim that $u \in H W_{0}^{1, Q}(\Omega)$. Indeed, for $\alpha>0$, let us define $u_{\alpha}(x)=N(x)^{\alpha} u(x)$. We note that $u_{\alpha} \in C_{0}(\Omega)$ for any $\alpha>0$. A simple computation show that

$$
\begin{aligned}
\nabla_{0} w(x)= & E_{1}^{-\frac{1+\alpha_{1}}{Q}}\left(\frac{N(x)}{R}\right) \prod_{k=2}^{m} E_{k}^{\frac{1-\alpha_{k}}{Q}}\left(\frac{N(x)}{R}\right) \\
& \times\left(\frac{Q-1-\alpha_{1}}{Q}+\sum_{k=2}^{m} \frac{1-\alpha_{i}}{Q} \frac{1}{\prod_{i=2}^{k} E_{i}\left(\frac{N(x)}{R}\right)}\right) \frac{\nabla_{0} N(x)}{N(x)},
\end{aligned}
$$

for a.e. $x \in \Omega$. Notice that $E_{i} \geq 1$ and there exists $C_{\alpha_{1}}>0$ such that

$$
\prod_{k=2}^{m} E_{i}^{\frac{1-\alpha_{i}}{Q}}(t) \leq C_{\alpha_{1}} E_{1}^{\frac{\alpha_{1}}{2 Q}}(t), \quad t \in(0,1] .
$$

Consequently, we have

$$
\left|\nabla_{0} w(x)\right| \leq \frac{Q+m-2}{Q} C_{\alpha_{1}} E_{1}^{-\frac{2+\alpha_{1}}{2 Q}}\left(\frac{N(x)}{R}\right) \frac{\left|\nabla_{0} N(x)\right|}{N(x)} .
$$

for a.e. $x \in \Omega$. Using the formulas (2.2) and (2.3), it is easy to see that

$$
\int_{B_{N} \backslash\{0\}}\left|\nabla_{0} w(x)\right|^{Q} d x<\infty
$$

Since

$$
\nabla u(x)=\psi^{\prime}\left(\frac{N(x)}{R}\right) w(x) \frac{\nabla_{0} N(x)}{R}+\varphi(x) \nabla_{0} w(x),
$$

for a.e. $x \in \Omega$ and the support of $\psi^{\prime}\left(\frac{N(x)}{R}\right)$ is contained in $\left\{x: \frac{1}{2} \leq N(x) \leq 1\right\}$, then we can readily check that

$$
\int_{\Omega \backslash\{0\}}\left|\nabla_{0} u(x)\right|^{Q} d x<\infty .
$$

Finally, we have

$$
\nabla_{o} u_{\alpha}(x)=\alpha N(x)^{\alpha-1} w(x) \nabla_{0} N(x)+N(x)^{\alpha} \nabla_{0} w(x),
$$

for a.e. $x \in \Omega$ and hence it is not hard to see that $\left|\nabla_{0} u_{\alpha}\right|^{Q} \in L^{1}(\Omega)$. Consequently, $u_{\alpha} \in H W_{0}^{1, Q}(\Omega)$. We show that

$$
\lim _{\alpha \rightarrow 0} \alpha^{Q} \int_{\Omega \backslash\{0\}} N(x)^{(\alpha-1) Q} w(x)^{Q}\left|\nabla_{0} N(x)\right|^{Q} d x=0 .
$$

Indeed, since $\varphi \leq 1$ and $E_{1} \geq 1$, we have

$$
\begin{aligned}
& \int_{\Omega \backslash\{0\}} N(x)^{(\alpha-1) Q} w(x)^{Q}\left|\nabla_{0} N(x)\right|^{Q} d x \\
& \leq C_{\alpha_{1}}^{Q} \int_{B_{N} \backslash\{0\}} N(x)^{(\alpha-1) Q} E_{1}^{Q-1-\frac{\alpha_{1}}{2}}\left(\frac{N(x)}{R}\right)\left|\nabla_{0} N(x)\right|^{Q} d x
\end{aligned}
$$

By (2.2) and (2.3), we get

$$
\int_{\Omega \backslash\{0\}} N(x)^{(\alpha-1) Q} w(x)^{Q}\left|\nabla_{0} N(x)\right|^{Q} d x \leq C_{\alpha_{1}}^{Q} c_{Q} \int_{0}^{1} t^{\alpha Q-1}\left(1-\ln \frac{t}{R}\right)^{Q-1-\frac{\alpha_{1}}{2}} d t .
$$


Making the change of variable $t=R e^{-s /(\alpha Q)}$, we obtain

$$
\int_{\Omega \backslash\{0\}} N(x)^{(\alpha-1) Q} w(x)^{Q}\left|\nabla_{0} N(x)\right|^{Q} d x \leq \frac{C_{\alpha_{1}}^{Q} c_{Q} R^{\alpha Q}}{(\alpha Q)^{Q-\frac{\alpha_{1}}{2}}} \int_{0}^{\infty} e^{-s}(\alpha Q+s)^{Q-1-\frac{\alpha_{1}}{2}} d s .
$$

Finally, we get

$\alpha^{Q} \int_{\Omega \backslash\{0\}} N(x)^{(\alpha-1) Q} w(x)^{Q}\left|\nabla_{0} N(x)\right|^{Q} d x \leq \frac{C_{\alpha_{1}}^{Q} c_{Q} R^{\alpha Q} \alpha^{\frac{\alpha_{1}}{2}}}{Q^{Q-\frac{\alpha_{1}}{2}}} \int_{0}^{\infty} e^{-s}(\alpha Q+s)^{Q-1-\frac{\alpha_{1}}{2}} d s$,

which then implies (3.4). On the other hand, by Lebesgue dominated convergence theorem we have

$$
\lim _{\alpha \rightarrow 0} \int_{\Omega \backslash\{0\}}\left|N(x)^{\alpha}-1\right|^{Q}\left|\nabla_{0} w(x)\right|^{Q} d x=0 .
$$

This limit together with (3.4) implies

$$
\lim _{\alpha \rightarrow 0} \int_{\Omega \backslash\{0\}}\left|\nabla_{0} u_{\alpha}(x)-\nabla_{0} u(x)\right|^{Q} d x=0 .
$$

Hence $u \in H W_{0}^{1, Q}(\Omega)$.

Using the elementary inequality

$$
|a+b|^{p} \leq|a|^{p}+C_{p}\left(|a|^{p-1}|b|+|b|^{p}\right), \quad a, b \in \mathbb{R}^{N}, N \geq 1, p \geq 2,
$$

for some constant $C_{p}$ depending only on $p$, we obtain

$$
\begin{aligned}
\int_{\Omega}\left|\nabla_{0} u(x)\right|^{Q} d x \leq & \int_{\Omega} \varphi(x)^{Q}\left|\nabla_{0} w(x)\right|^{Q} d x \\
& +C_{Q} \int_{\Omega}\left|\nabla_{0} \varphi(x)\right| \varphi(x)^{Q-1}\left|\nabla_{0} w(x)\right|^{Q-1} w(x) d x \\
& +C_{Q} \int_{\Omega}\left|\nabla_{0} \varphi(x)\right|^{Q} w(x)^{Q} d x=I_{1}+I_{2}+I_{3} .
\end{aligned}
$$

It is not hard to see that $I_{2}$ and $I_{3}$ are uniformly bounded with respect to the $\alpha_{1}, \ldots, \alpha_{m}$. Hence

$$
\int_{\Omega}\left|\nabla_{0} u(x)\right|^{Q} d x \leq \int_{\Omega} \varphi(x)^{Q}\left|\nabla_{0} w(x)\right|^{Q} d x+O(1)
$$

uniformly as $\alpha_{1}, \ldots, \alpha_{m} \rightarrow 0$.

From the computation above, we have

$$
\nabla_{0} w(x)=E_{1}^{-\frac{1+\alpha_{1}}{Q}}\left(\frac{N(x)}{R}\right) \prod_{k=2}^{m} E_{k}^{\frac{1-\alpha_{k}}{Q}}\left(\frac{N(x)}{R}\right)\left(\frac{Q-1}{Q}+\frac{\eta(x)}{Q}\right) \frac{\nabla_{0} N(x)}{N(x)},
$$

for a.e. $x \in \Omega$ with

$$
\eta(x)=-\alpha_{1}+\sum_{k=2}^{m}\left(1-\alpha_{i}\right) \frac{1}{\prod_{i=2}^{k} E_{i}\left(\frac{N(x)}{R}\right)} .
$$

Notice that $\eta(x)$ is uniformly bounded as $\alpha_{1}, \ldots, \alpha_{m} \rightarrow 0$, hence there exists a positive constant $c$ such that

$$
\left|\frac{Q-1}{Q}+\frac{\eta(x)}{Q}\right|^{Q} \leq\left(\frac{Q-1}{Q}\right)^{Q}+\left(\frac{Q-1}{Q}\right)^{Q-1} \eta+\frac{1}{2}\left(\frac{Q-1}{Q}\right)^{Q-1} \eta^{2}+c|\eta|^{3} .
$$


So we have

$$
\begin{aligned}
& \int_{\Omega} \varphi(x)^{Q}\left|\nabla_{0} w(x)\right|^{Q} d x \leq \int_{\Omega} \varphi(x)^{Q} E_{1}^{-1-\alpha_{1}}\left(\frac{N(x)}{R}\right) \prod_{k=2}^{m} E_{k}^{1-\alpha_{k}}\left(\frac{N(x)}{R}\right) \\
& \times\left(\left(\frac{Q-1}{Q}\right)^{Q}+\left(\frac{Q-1}{Q}\right)^{Q-1} \eta+\frac{1}{2}\left(\frac{Q-1}{Q}\right)^{Q-1} \eta^{2}+c|\eta|^{3}\right) \frac{\left|\nabla_{0} N(x)\right|^{Q}}{N(x)^{Q}} d x \\
(3.6) & =J_{1}+J_{2}+J_{3}+J_{4} .
\end{aligned}
$$

Since $E_{i} \geq 1$, then there exists a positive constant $c^{\prime}$ such that

$$
|\eta|^{3} \leq c^{\prime}\left(\alpha_{1}^{3}+\frac{1}{E_{2}^{3}\left(\frac{N(x)}{R}\right)}\right) .
$$

Furthermore, there exists $c^{\prime \prime}>0$ such that

$$
\prod_{k=2}^{m} E_{k}^{1-\alpha_{k}} \leq c^{\prime \prime} E_{2}^{\frac{3}{2}}
$$

Therefore

$$
\begin{aligned}
J_{4} & \leq c c^{\prime} c^{\prime \prime} \int_{B_{N}} E_{1}^{-1-\alpha_{1}}\left(\frac{N(x)}{R}\right) E_{2}^{\frac{3}{2}}\left(\frac{N(x)}{R}\right)\left(\alpha_{1}^{3}+\frac{1}{E_{2}^{3}\left(\frac{N(x)}{R}\right)}\right) \frac{\left|\nabla_{0} N(x)\right|^{Q}}{N(x)^{Q}} d x \\
& =c c^{\prime} c^{\prime \prime}\left(\alpha_{1}^{3} J_{41}+J_{42}\right) .
\end{aligned}
$$

By (2.2) and (2.3), we have

$$
J_{41}=c_{Q} \int_{0}^{1} E_{1}^{-1-\alpha_{1}}\left(\frac{t}{R}\right) E_{2}^{\frac{3}{2}}\left(\frac{t}{R}\right) \frac{d t}{t} .
$$

Making the change of variable $s=\alpha_{1} E_{2}(t / R)$, we get

$$
J_{41} \leq \alpha_{1}^{-\frac{5}{2}} e^{\alpha_{1}} c_{Q} \int_{0}^{\infty} e^{-s} s^{\frac{3}{2}} d s .
$$

Hence $\alpha_{1}^{3} J_{41}=O(1)$ uniformly as $\alpha_{1}, \ldots, \alpha_{m} \rightarrow 0$. Similarly, we have

$$
J_{42} \leq c_{Q} \int_{E_{2}(1 / R)}^{\infty} e^{-\alpha_{1}(s-1)} s^{-\frac{3}{2}} d s=O(1),
$$

uniformly as $\alpha_{1}, \ldots, \alpha_{m} \rightarrow 0$. Thus, we have proved

$$
J_{4}=O(1), \quad \text { uniformly as } \alpha_{1}, \ldots, \alpha_{m} \rightarrow 0 .
$$

Notice that

$$
J_{1}=\left(\frac{Q-1}{Q}\right)^{Q} \int_{\Omega} \frac{|u(x)|^{Q}}{N(x)^{Q} E_{1}^{Q}\left(\frac{N(x)}{R}\right)}\left|\nabla_{0} N(x)\right|^{Q} d x
$$

Denote

$$
\begin{aligned}
I_{m-1}:= & \int_{\Omega}\left|\nabla_{0} u\right|^{Q} d x-\left(\frac{Q-1}{Q}\right)^{Q} \int_{\Omega}\left|\nabla_{0} N(x)\right|^{Q} \frac{|u(x)|^{Q}}{N(x)^{Q} E_{1}^{Q}\left(\frac{N(x)}{R}\right)^{Q}} d x \\
& -\frac{1}{2}\left(\frac{Q-1}{Q}\right)^{Q-1} \sum_{k=2}^{m-1} \int_{\Omega}\left|\nabla_{0} N(x)\right|^{Q} \frac{|u(x)|^{Q}}{N(x)^{Q} E_{1}^{Q}\left(\frac{N(x)}{R}\right) \prod_{i=2}^{k} E_{i}^{2}\left(\frac{N(x)}{R}\right)} d x .
\end{aligned}
$$


Expanding $\eta^{2}$, collecting the similar terms and using (3.5) and (3.6), we have

$$
I_{m-1} \leq \frac{1}{2}\left(\frac{Q-1}{Q}\right)^{Q-1} J+O(1)
$$

with

$$
J=A_{m}+\sum_{i=1}^{m}\left(\alpha_{i}^{2}-2 \alpha_{i}\right) A_{i}+2 \sum_{i=1}^{m-1} \sum_{j=i+1}^{m}\left(1-\alpha_{i}\right)\left(1-\alpha_{j}\right) B_{i j}
$$

where

$$
\begin{aligned}
& A_{1}=\int_{\Omega} \varphi(x)^{Q} E_{1}^{-1-\alpha_{1}}\left(\frac{N(x)}{R}\right) \prod_{k=2}^{m} E_{k}^{1-\alpha_{k}}\left(\frac{N(x)}{R}\right) \frac{\left|\nabla_{0} N(x)\right|^{Q}}{N(x)^{Q}} d x \\
& A_{i}=\int_{\Omega} \varphi(x)^{Q} E_{1}^{-1-\alpha_{1}}\left(\frac{N(x)}{R}\right) \prod_{k=2}^{i} E_{k}^{-1-\alpha_{k}}\left(\frac{N(x)}{R}\right) \prod_{k=i+1}^{m} E_{k}^{1-\alpha_{k}}\left(\frac{N(x)}{R}\right) \frac{\left|\nabla_{0} N(x)\right|^{Q}}{N(x)^{Q}} d x
\end{aligned}
$$

for $2 \leq i \leq m$,

$$
B_{1, j}=\int_{\Omega} \varphi(x)^{Q} E_{1}^{-1-\alpha_{1}}\left(\frac{N(x)}{R}\right) \prod_{k=2}^{j} E_{k}^{-\alpha_{k}}\left(\frac{N(x)}{R}\right) \prod_{k=j+1}^{m} E_{k}^{1-\alpha_{k}}\left(\frac{N(x)}{R}\right) \frac{\left|\nabla_{0} N(x)\right|^{Q}}{N(x)^{Q}} d x
$$

for $2 \leq j \leq m$, and

$$
\begin{aligned}
B_{i j}= & \int_{\Omega} \varphi(x)^{Q} E_{1}^{-1-\alpha_{1}}\left(\frac{N(x)}{R}\right) \prod_{k=2}^{i} E_{k}^{-1-\alpha_{k}}\left(\frac{N(x)}{R}\right) \prod_{k=i+1}^{j} E_{k}^{-\alpha_{k}}\left(\frac{N(x)}{R}\right) \\
& \times \prod_{k=j+1}^{m} E_{k}^{1-\alpha_{k}}\left(\frac{N(x)}{R}\right) \frac{\left|\nabla_{0} N(x)\right|^{Q}}{N(x)^{Q}} d x
\end{aligned}
$$

for $2 \leq i<j \leq m$.

We intend to take the limit $\alpha_{1} \rightarrow 0$ in (3.8). By using the similar estimate for $J_{41}$ and $J_{42}$ we see that all terms in (3.8) has finite limit except $A_{1}$ and $B_{1 j}$. We have by $(2.2),(2.3)$ and integration by parts that

$$
\begin{aligned}
\alpha_{1} A_{1} & =c_{Q} \alpha_{1} \int_{0}^{1} \psi(t)^{Q} E_{1}^{-1-\alpha_{1}}\left(\frac{t}{R}\right) \prod_{k=2}^{m} E_{k}^{1-\alpha_{k}}\left(\frac{t}{R}\right) t^{-1} d t \\
& =c_{Q} \int_{0}^{1} \psi(t)^{Q}\left(E_{1}^{-\alpha_{1}}\left(\frac{t}{R}\right)\right)^{\prime} \prod_{k=2}^{m} E_{k}^{1-\alpha_{k}}\left(\frac{t}{R}\right) d t \\
& =\sum_{j=2}^{m}\left(1-\alpha_{j}\right) B_{1 j}-Q c_{Q} \int_{0}^{1} \psi^{\prime}(t) E_{1}^{-\alpha_{1}}\left(\frac{t}{R}\right) \prod_{k=2}^{m} E_{k}^{1-\alpha_{k}}\left(\frac{t}{R}\right) d t .
\end{aligned}
$$

The second term on the right-hand side is $O(1)$. Similarly, we get

$$
B_{1 j}=-\sum_{i=2}^{j-1} \alpha_{i} B_{i j}-\alpha_{j} A_{j}+\sum_{i=j+1}^{m}\left(1-\alpha_{i}\right) B_{j i}+O(1)
$$


Consequently, we have

$$
\begin{aligned}
& \left(\alpha_{1}^{2}-2 \alpha_{1}\right) A_{1}+2 \sum_{j=2}^{m}\left(1-\alpha_{1}\right)\left(1-\alpha_{j}\right) B_{1 j} \\
& =\sum_{i=2}^{m}\left(\alpha_{i}-\alpha_{i}^{2}\right) A_{i}+\sum_{i=2}^{m-1} \sum_{j=i+1}^{m}\left(2 \alpha_{i}-1\right)\left(1-\alpha_{j}\right) B_{i j}+O(1) .
\end{aligned}
$$

Inserting this expression into (3.8) and letting $\alpha_{1} \rightarrow 0$, we get

$$
J=A_{m}-\sum_{i=2}^{m} \alpha_{i} A_{i}+\sum_{i=2}^{m-1} \sum_{j=i+1}^{m}\left(1-\alpha_{j}\right) B_{i j}+O(1), \quad\left(\alpha_{1}=0\right) .
$$

Similarly, we intend to take the limit $\alpha_{2} \rightarrow 0$. We have by (2.2), (2.3) and integration by parts that

$$
\begin{aligned}
\alpha_{2} A_{2} & =c_{Q} \alpha_{2} \int_{0}^{1} \psi(t)^{Q} E_{1}^{-1}\left(\frac{t}{R}\right) E_{2}^{-1-\alpha_{k}}\left(\frac{t}{R}\right) \prod_{k=3}^{m} E_{k}^{1-\alpha_{k}}\left(\frac{t}{R}\right) t^{-1} d t \\
& =c_{Q} \int_{0}^{1} \psi(t)^{Q}\left(E_{2}^{-\alpha_{2}}\left(\frac{t}{R}\right)\right)^{\prime} \prod_{k=3}^{m} E_{k}^{1-\alpha_{k}}\left(\frac{t}{R}\right) d t \\
& =\sum_{j=3}^{m}\left(1-\alpha_{j}\right) B_{2 j}-Q c_{Q} \int_{0}^{1} \psi^{\prime}(t) E_{2}^{-\alpha_{2}}\left(\frac{t}{R}\right) \prod_{k=3}^{m} E_{k}^{1-\alpha_{k}}\left(\frac{t}{R}\right) d t .
\end{aligned}
$$

The second term on the right-hand side is $O(1)$. Inserting the previous expression into (3.9) and letting $\alpha_{2} \rightarrow 0$, we get

$$
J=A_{m}-\sum_{i=3}^{m} \alpha_{i} A_{i}+\sum_{i=3}^{m-1} \sum_{j=i+1}^{m}\left(1-\alpha_{j}\right) B_{i j}+O(1), \quad\left(\alpha_{1}=\alpha_{2}=0\right) .
$$

Repeating this process, we arrive

$$
J=\left(1-\alpha_{m}\right) A_{m}+O(1), \quad\left(\alpha_{1}=\alpha_{2}=\cdots=\alpha_{m-1}=0\right) .
$$

Combining (1.5), (3.7) and (3.10) together, we get

$$
B \leq \frac{1}{2}\left(\frac{Q-1}{Q}\right)^{Q-1} \frac{\left(1-\alpha_{m}\right) A_{m}+O(1)}{\int_{\Omega} \frac{\varphi(x)^{Q}\left|\nabla_{0} N(x)\right|^{Q}}{N(x)^{Q} E_{1}\left(\frac{N(x)}{R}\right) \prod_{i=2}^{m-1} E_{i}\left(\frac{N(x)}{R}\right) E_{m}^{\gamma-1+\alpha_{m}}\left(\frac{N(x)}{R}\right)} d x} .
$$

We have

$$
\begin{aligned}
A_{m} & =c_{Q} \int_{0}^{1} \psi(t)^{Q} \frac{1}{t \prod_{i=1}^{m-1} E_{i}\left(\frac{t}{R}\right) E_{m}^{1+\alpha_{m}}\left(\frac{t}{R}\right)} d t \\
& =c_{Q} \int_{E_{m}(1 / R)}^{\infty} s^{-1-\alpha_{m}} d t \quad\left(s=E_{m}(t / R)\right) \\
& =\frac{c_{Q}}{\alpha_{m}} E_{m}^{-\alpha_{m}}\left(\frac{1}{R}\right) .
\end{aligned}
$$

Hence $A_{m} \rightarrow \infty$ as $\alpha_{m} \rightarrow 0$. Since $B>0$, we then have

$$
\int_{\Omega} \frac{\varphi(x)^{Q}\left|\nabla_{0} N(x)\right|^{Q}}{N(x)^{Q} E_{1}\left(\frac{N(x)}{R}\right) \prod_{i=2}^{m-1} E_{i}\left(\frac{N(x)}{R}\right) E_{m}^{\gamma-1+\alpha_{m}}\left(\frac{N(x)}{R}\right)} d x<\infty,
$$


for any $\alpha_{m}>0$ small. On the other hand, we have

$$
\begin{aligned}
& \int_{\Omega} \varphi(x)^{Q}\left|\nabla_{0} N(x)\right|^{Q} \frac{1}{N(x)^{Q} E_{1}\left(\frac{N(x)}{R}\right) \prod_{i=2}^{m-1} E_{i}\left(\frac{N(x)}{R}\right) E_{m}^{\gamma-1+\alpha_{m}}\left(\frac{N(x)}{R}\right)} d x \\
& =c_{Q} \int_{0}^{1} \psi(t) \frac{1}{t E_{1}\left(\frac{t}{R}\right) \prod_{i=2}^{m-1} E_{i}\left(\frac{t}{R}\right) E_{m}^{\gamma-1+\alpha_{m}}\left(\frac{t}{R}\right)} d t \\
& =c_{Q} \int_{E_{m}(1 / R)}^{\infty} s^{1-\gamma-\alpha_{m}} d s \quad\left(s=E_{m}(t / R)\right) .
\end{aligned}
$$

The preceding integral is finite if and only if $\alpha_{m}>2-\gamma$. Thus we have $\gamma \geq 2-\alpha_{m}$ for $\alpha_{m}>0$ small. Letting $\alpha_{m} \downarrow 0$, we obtain $\gamma \geq 2$.

Suppose $\gamma=2$, then we have

$$
B \leq \frac{1}{2}\left(\frac{Q-1}{Q}\right)^{Q-1}\left(1-\alpha_{m}+O\left(A_{m}^{-1}\right)\right) .
$$

Letting $\alpha_{m} \rightarrow 0$ and using the fact $A_{m} \rightarrow \infty$, we obtain $B \leq \frac{1}{2}\left(\frac{Q-1}{Q}\right)^{Q-1}$ as desired.

This theorem is completely proved.

\section{Proof of Theorem 1.2}

In this section, we prove Theorem 1.2. The proof is based on the improved critical Hardy inequality from Theorem 1.1 and the Moser-Trudinger inequality in Carnot groups from [8].

Proof of Theorem 1.2. By density argument, it is enough to prove Theorem 1.2 for functions in $C_{0}^{\infty}(\Omega \backslash\{0\})$ with $I_{G}[u, \Omega, R] \leq 1$. Let $v(x)=E_{1}^{\frac{1-Q}{Q}}\left(\frac{N(x)}{R}\right) u(x)$, $x \in G$. We note that

$$
\begin{aligned}
\nabla_{0}\left(\frac{u(x)}{E_{2}^{\frac{2}{Q}}\left(\frac{N(x)}{R}\right)}\right) & =\nabla_{0}\left(\frac{v(x) E_{1}^{\frac{Q-1}{Q}}\left(\frac{N(x)}{R}\right)}{E_{2}^{\frac{2}{Q}}\left(\frac{N(x)}{R}\right)}\right) \\
& =\nabla_{0} v(x) \frac{E_{1}^{\frac{Q-1}{Q}}\left(\frac{N(x)}{R}\right)}{E_{2}^{\frac{2}{Q}}\left(\frac{N(x)}{R}\right)}+v(x) \nabla_{0}\left(\frac{E_{1}^{\frac{Q-1}{Q}}\left(\frac{N(x)}{R}\right)}{E_{2}^{\frac{2}{Q}}\left(\frac{N(x)}{R}\right)}\right) .
\end{aligned}
$$

The direct calculations show that

$$
\nabla_{0}\left(\frac{E_{1}^{\frac{Q-1}{Q}}\left(\frac{N(y)}{R}\right)}{E_{2}^{\frac{2}{Q}}\left(\frac{N(y)}{R}\right)}\right)=-\frac{\nabla_{0} N(y)}{N(y)} \frac{1}{E_{1}^{\frac{1}{Q}}\left(\frac{N(y)}{R}\right) E_{2}^{\frac{2}{Q}}\left(\frac{N(y)}{R}\right)}\left(\frac{Q-1}{Q}-\frac{2}{Q E_{2}\left(\frac{N(y)}{R}\right)}\right) .
$$

Since $Q \geq 3$ and $E_{2} \geq 1$, we then have

$$
\left|\nabla_{0}\left(\frac{E_{1}^{\frac{Q-1}{Q}}\left(\frac{N(y)}{R}\right)}{E_{2}^{\frac{2}{Q}}\left(\frac{N(y)}{R}\right)}\right)\right| \leq \frac{Q-1}{Q} \frac{\left|\nabla_{0} N(y)\right|}{N(y)} \frac{1}{E_{1}^{\frac{1}{Q}}\left(\frac{N(y)}{R}\right) E_{2}^{\frac{2}{Q}}\left(\frac{N(y)}{R}\right)} .
$$


Using again $E_{2} \geq 1$ and $u(x)=v(x) E_{1}^{\frac{Q-1}{Q}}\left(\frac{N(x)}{R}\right)$, we get

$$
\begin{aligned}
& \left|\nabla_{0}\left(\frac{u(x)}{E_{2}^{\frac{2}{Q}}\left(\frac{N(x)}{R}\right)}\right)\right| \\
& \leq\left|\nabla_{0} v(x)\right| E_{1}^{\frac{Q-1}{Q}}\left(\frac{N(x)}{R}\right)+\frac{Q-1}{Q} \frac{\left|\nabla_{0} N(x)\right|}{N(x)} \frac{|u(x)|}{E_{1}\left(\frac{N(x)}{R}\right) E_{2}^{\frac{2}{Q}}\left(\frac{N(x)}{R}\right)} .
\end{aligned}
$$

Theorem 1.1 and Proposition 2.1 yield

$$
\left\|\nabla_{0}\left(\frac{u(x)}{E_{2}^{\frac{2}{Q}}\left(\frac{N(x)}{R}\right)}\right)\right\|_{L^{Q}(\Omega)}^{Q} \leq 2^{Q-1}\left(C_{1}(Q)+2 \frac{Q}{Q-1}\right)=: b_{Q}^{Q},
$$

here we use the convexity inequality $|a+b|^{Q} \leq 2^{Q-1}\left(|a|^{Q}+|b|^{Q}\right)$. Define $A_{Q, N}=$ $b_{Q}^{-\frac{Q}{Q-1}} A_{Q}$. Then the inequality (1.10) follows from (1.7) and (4.1).

For the second statement, we prove by contradiction argument. By scaling argument, we can assume that the unit ball $B_{N}$ is included in $\Omega$. Suppose that there exists $c>0$ so that (1.10) holds for $\beta<\frac{1}{Q}$ and $R \geq R_{\Omega}$. Choose $\theta \in(1,2)$ such that $1<Q \beta+\theta<2$. By repeating the argument in the proof of [36, Theorem 1.1], we would get the following inequality

$$
I_{G}[u, \Omega, R] \geq C \int_{\Omega} \frac{|u(x)|^{Q}}{N(x)^{Q} E_{1}^{Q}\left(\frac{N(x)}{R}\right) E_{2}^{Q \beta+\theta}\left(\frac{N(x)}{R}\right)}\left|\nabla_{0} N(x)\right|^{Q} d x, \quad u \in H W_{0}^{1, Q}(\Omega)
$$

for some $C>0$ which violates Theorem 1.1 since $Q \beta+\theta<2$. This contradiction finishes the proof of the second statement.

\section{References}

[1] Adams, D. R.: A sharp inequality of J. Moser for higher order derivatives. - Ann. of Math. (2) $128: 2,1988,385-398$.

[2] Adimurthi, N. Chaudhuri, and M. Ramaswamy: An improved Hardy-Sobolev inequality and its application. - Proc. Amer. Math. Soc. 130:2, 2002, 489-505.

[3] Adimurthi, and O. Druet: Blow-up analysis in dimension 2 and a sharp form of TrudingerMoser inequality. - Comm. Partial Differential Equations 29:1-2, 2004, 295-322.

[4] Adimurthi, and K. Sandeep: Existence and non-existence of the first eigenvalue of the perturbed Hardy-Sobolev operator. - Proc. Roy. Soc. Edinburgh Sect. A 132:5, 2002, 10211043.

[5] Adimurthi, and K. SandeeP: A singular Moser-Trudinger embedding and its applications. NoDEA Nonlinear Differential Equations Appl. 13:5-6, 2007, 585-603.

[6] Balogh, Z. M., I. Holopainen, and J. T. Tyson: Singular solutions, homogeneous norms, and quasiconformal mappings in Carnot groups. - Math. Ann. 324:1, 2002, 159-186.

[7] Balogh, Z. M., and J. T. Tyson: Polar coordinates in Carnot groups. - Math. Z. 241:4, 2002, 697-730.

[8] Balogh, Z. M., J. J. Manfredi, and J. T. Tyson: Fundamental solution for the $Q$-Laplacian and sharp Moser-Trudinger inequality in Carnot groups. - J. Funct. Anal. 204:1, 2003, 35-49.

[9] Barbatis, G., S. Filippas, and A. Tertikas: Series expansion for $L^{p}$ Hardy inequalities Indiana Univ. Math. J. 52:1, 2003, 171-190. 
[10] Barbatis, G., S. Filippas, and A. Tertikas: A unified approach to improved $L^{p}$ Hardy inequalities with best constants. - Trans. Amer. Math. Soc. 356:6, 2004, 2169-2196.

[11] Brezis, H., and J.-L. VÁzQuez: Blow-up solutions of some nonlinear elliptic problems. - Rev. Mat. Univ. Comp. Madrid 10, 1997, 443-469.

[12] Capogna, L., D. Danielli, and N. Garofalo: Capacitary estimates and the local behavior of solutions of nonlinear subelliptic equations. - Amer. J. Math. 118:6, 1996, 1153-1196.

[13] Carleson, L., and A. Chang: On the existence of an extremal function for an inequality of J. Moser. - Bull. Sci. Math. 110, 1986, 113-127.

[14] Cohn, W. S., N. LAM, G. Lu, and Y. YANG: The Moser-Trudinger inequality in unbounded domains of Heisenberg group and sub-elliptic equations. - Nonlinear Anal. 75:12, 2012, 44834495 .

[15] Cohn, W. S., and G. Lu: Best constants for Moser-Trudinger inequalities on the Heisenberg group. - Indiana Univ. Math. J. 50:4, 2001, 1567-1591.

[16] Cohn, W. S., and G. LU: Best constants of Moser-Trudinger inequalities, fundamental solutions and one-parameter representation formulas on groups of Heisenberg type. - Acta Math. Sin. (Engl. Ser.) 18:2, 2002, 375-390.

[17] Csató, G., V.H. NGuyen, and P. Roy: Extremals for the singular Moser-Trudinger inequality via $n$-harmonic transplantation. - J. Differential Equations 270, 2021, 843-882.

[18] Danielli, D., N. Garofalo, and N.C. PhuC: Hardy-Sobolev type inequalities with sharp constants in Carnot-Carathéodory spaces. - Potential Anal. 34:3, 2011, 223-242.

[19] Dou, J., P. Niu, and Z. YuAn: A Hardy inequality with remainder terms in the Heisenberg group and the weighted eigenvalue problem. - J. Inequal. Appl. 2007, Art. ID 32585, 1-24.

[20] Filippas, S., and A. Tertikas: Optimizing improved Hardy inequalities. - J. Funct. Anal. $192,2002,186-233$.

[21] Flucher, M.: Extremal functions for Trudinger-Moser inequality in 2 dimensions. - Comment. Math. Helv. 67, 1992, 471-497.

[22] Folland, G. B.: Subelliptic estimates and function spaces on nilpotent Lie groups. - Ark. Mat. 13:2, 1975, 161-207.

[23] Folland, G. B., and E. M. Stein: Hardy spaces on homogeneous groups. - Princeton Univ. Press, Princeton, NJ, 1982.

[24] Garofalo, N., and E. Lanconelli: Frequency functions on the Heisenberg group, the uncertainty principle and unique continuation. - Ann. Inst. Fourier (Grenoble) 40:2, 1990, 313-356.

[25] Gazzola, F., H. C. Grunau, and E. Mitidieri: Hardy inequalities with optimal constants and remainder terms. - Trans. Amer. Math. Soc. 356, 2004, 2149-2168.

[26] Goldstein, J. A., and I. Kombe: The Hardy inequality and nonlinear parabolic equations on Carnot groups.- Nonlinear Anal. 69:12, 2008, 4643-4653.

[27] Goldstein, J. A., I. Kombe, and A. Yener: A unified approach to weighted Hardy type inequalities on Carnot groups. - Discrete Contin. Dyn. Syst. 37:4, 2017, 2009-2021.

[28] Heinonen, J., and I. Holopainen: Quasiregular maps on Carnot groups. - J. Geom. Anal. 7:1, 1997, 109-148.

[29] LAm, N., and G. Lu: Sharp Moser-Trudinger inequality on the Heisenberg group at the critical case and applications. - Adv. Math. 231:6, 2012, 3259-3287.

[30] LAm, N., and G. Lu: A new approach to sharp Moser-Trudinger and Adams type inequalities: a rearrangement-free argument. - J. Differential Equations 255:3, 2013, 298-325.

[31] LAm, N., and H. TANG: Sharp constants for weighted Moser-Trudinger inequalities on groups of Heisenberg type. - Nonlinear Anal. 89, 2013, 95-109.

[32] LeRAY, J.: Étude de diverses équations intégrales non linéaires et de quelques problèmes que pose l'Hydrodynamique. - J. Math. Pures Appl. 12, 1933, 1-82. 
[33] Li, Y., and B. RuF: A sharp Trudinger-Moser type inequality for unbounded domains in $\mathbb{R}^{n}$. - Indiana Univ. Math. J. 57, 2008, 451-480.

[34] Lin, K. C.: Extremal functions for Moser's inequality. - Trans. Amer. Math. Soc. 348, 1996, 2663-2671.

[35] Mancini, G., and K. Sandeep: Moser-Trudinger inequality on conformal disks. - Commun. Contemp. Math. 12:6, 2010, 1055-1068.

[36] Mallick, A., and C. Tintarev: An improved Leray-Trudinger inequality. - Commun. Contemp. Math. 20:4, 2018, 1750034, 1-14.

[37] Moser, J.: A sharp form of an inequality by N. Trudinger. - Indiana Univ. Math. J. 20, 1970/71, 1077-1092.

[38] Nguyen, V.H.: The Leray-Adams inequality. - Preprint, arXiv:1902.10970.

[39] O'NeIL, R.: Convolution operators and $L(p, q)$ spaces. - Duke Math. J. 30, 1963, 129-142.

[40] PohožAev, S. I.: On the eigenfunctions of the equation $\Delta u+\lambda f(u)=0$. - Dokl. Akad. Nauk. SSSR 165, 1965, 36-39 (in Russian).

[41] Psaradakis, G., and D. Spector: A Leray-Trudinger inequality. - J. Funct. Anal. 269, 2015, 215-228.

[42] Ruf, B.: A sharp Trudinger-Moser type inequality for unbounded domains in $\mathbb{R}^{2}$. - J. Funct. Anal. 219, 2005, 340-367.

[43] Ruzhansky, M., and N. Yessirkegenov: New progress on weighted Trudinger-Moser and Gagliardo-Nirenberg, and critical Hardy inequalities on stratified groups. - In: Landscapes of time-frequency analysis, edited by P. Boggiatto et al., Applied and Numerical Harmonic Analysis, Birkhäuser, 2019.

[44] Ruzhansky, M., and N. Yessirkegenov: Critical Sobolev, Gagliardo-Nirenberg, Trudinger and Brezis-Gallouet-Wainger inequalities, best constants, and ground states on graded groups. - Preprint, arXiv:1709.08263.

[45] Ruzhansky, M., and N. Yessirkegenov: Hypoelliptic functional inequalities. - Preprint, arXiv:1805.01064.

[46] Saloff-Coste, L., Théorèmes de Sobolev et inégalités de Trudinger sur certains groupes de Lie. - C. R. Acad. Sci. Paris Sér. I Math. 306:6, 1988, 305-308.

[47] Tintarev, C.: Trudinger-Moser inequality with remainder terms. - J. Funct. Anal. 266, 2014, 55-66.

[48] Trudinger, N. S.: On imbedding into Orlicz spaces and some applications. - J. Math. Mech. 17, 1967, 473-483.

[49] Tyson, J.T.: Sharp weighted Young's inequalities and Moser-Trudinger inequalities on Heisenberg type groups and Grushin spaces. - Potential Anal. 24, 2006, 357-384.

[50] Yudovič, V. I.: Some estimates connected with integral operators and with solutions of elliptic equations. - Dokl. Akad. Nauk. SSSR 138, 1961, 805-808 (in Russian).

Received 1 February 2020 • Accepted 25 January $2021 \bullet$ Published online 2 December 2021

\author{
Van Hoang Nguyen \\ FPT University \\ Department of Mathematics \\ Ha Noi, Vietnam \\ hoangnv47@fe.edu.vn
}

